\title{
Editorial
}

J. Synchrotron Rad. (1997). 4, 315

\section{Fifty Years of Synchrotron Radiation}

\author{
S. Samar Hasnain, ${ }^{a}$ John R. Helliwell ${ }^{b}$ and Hiromichi Kamitsubo ${ }^{c}$ \\ ${ }^{a}$ CCLRC Daresbury Laboratory, Warrington WA4 4AD, UK, ${ }^{b}$ Department of Chemistry, \\ Manchester University, Manchester M13 9PL, UK, and ' SPring-8, JAERI-RIKEN, 2-28-8 \\ Honkomagome, Bunkyo-ku, Tokyo 113, Japan
}

This issue marks the 50th anniversary of the first observation of synchrotron radiation light from the General Electric $70 \mathrm{MeV}$ synchrotron and coincides with the third anniversary of the Journal of Synchrotron Radiation (JSR). The issue brings together review articles from some of the pioneers of the field and in our view forms a unique historical record.

The interdisciplinary nature of synchrotron radiation research has been apparent from the early 1970's and the planners of the second- and third-generation sources have paid particular attention to the requirements of such an interdisciplinary community. It is clear that synchrotron radiation has removed the boundaries in otherwise somewhat isolated research areas. Indeed, JSR is playing an important part in furthering such interdisciplinary interactions. Over the last three years we have brought together the broad synchrotron radiation community by offering rapid communication of scientific and technical developments in the journal. We have not compromised on the quality of the contributions and have managed to combine this with a speed and high standard of production. In this period, more than half a dozen third-generation synchrotron radiation sources have come into operation and several more are now at an advanced state of planning or construction (e.g. SLS, DIAMOND and SOLEIL). Worldwide this is still a rapidly expanding field.

It is now just over 40 years since the first synchrotron radiation experiments were performed by Tomboulin and Hartman at Cornell in the soft X-ray region. These experiments were immediately followed by those of Madden and Codling in 1961 at the National Bureau of Standards in the UV/VUV region. This early utilization demonstrated the wealth of physics that needed to be explored by these new continuum light sources. Initiatives in Europe (Frascati, DESY, NINA), the former Soviet Union (VEPP) and Japan (INS-SOR) began in the mid-1960's along with expanding scientific opportunities. The first biological experiments were performed at DESY by Holmes, Rosenbaum and Witz in 1970 , opening synchrotron radiation to the biological community.

We again reiterate the main objectives of $J S R$; namely, to provide a focus for the whole of the Synchrotron Radiation community, and to accept papers only of a high standard covering sources, instrumentation, methods and applications. Through 1997 our main aim has been to increase the size of each issue. We are grateful to the many authors who have responded enthusiastically to our call. We continue to urge you to always consider $J S R$ as the first choice when you have new results, methods or instrumental facilities. It is only through your high-quality contributions that our community will be able to sustain a high-quality journal.

We accompany this editorial with a photograph (Fig. 1) taken in August 1997 at the Synchrotron Radiation Instrumentation Conference in Himeji, Japan, featuring some of the pioneers of the synchrotron radiation field (papers from the conference will be published in the May 1998 issue of the journal).

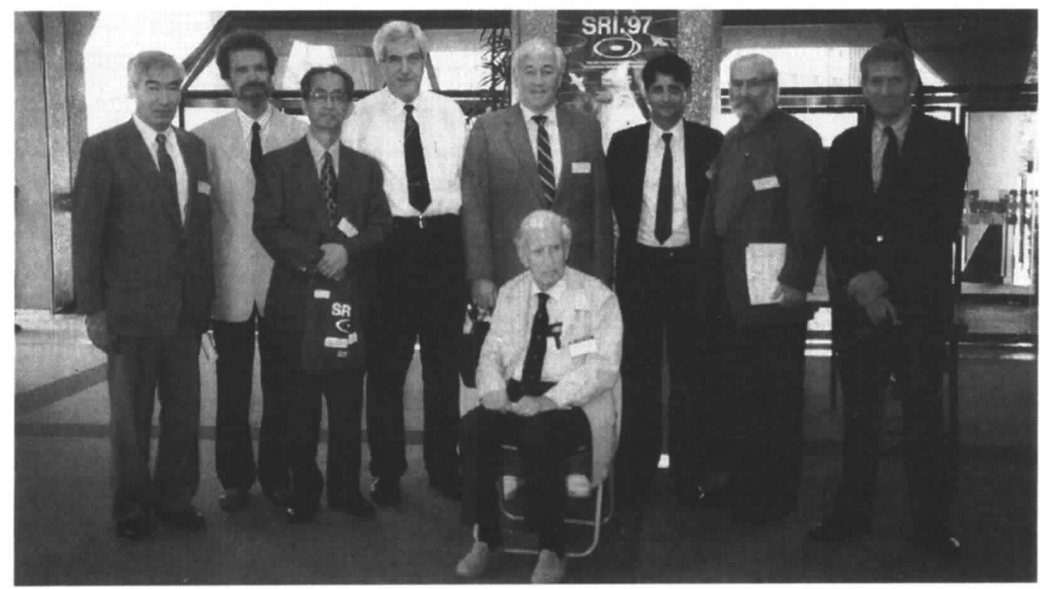

(C) 1997 International Union of Crystallography

Printed in Great Britain - all rights reserved

\section{Figure 1}

Left to right: H. Kamitsubo, G. Rosenbaum, T. Sasaki, R. Haensel, R. P. Madden, S. S. Hasnain, H. Winick and K. C. Holmes, with J. P. Blewett sitting in front, who had predicted and observed the shrinkage of electron orbit due to synchrotron radiation loss in 1945; see his review in the May 1998 issue. 